MODELING, IDENTIFICATION AND CONTROL, 1994, VOL. 15, NO. 2, 65-79

doi:10.4173/mic.1994.21

\title{
Ride control of surface effect ships using distributed control
}

\author{
A. J. SØRENSEN† and O. EGELAND $\$$
}

Keywords: SES, acoustic, ride control, passivity, full scale results

A ride control system for active damping of heave and pitch accelerations of Surface Effect Ships (SES) is presented. It is demonstrated that distributed effects that are due to a spatially varying pressure in the air cushion result in significant vertical vibrations in low and moderate sea states. In order to achieve a high quality human comfort and crew workability it is necessary to reduce these vibrations using a control system which accounts for distributed effects due to spatial pressure variations in the air cushion. A mathematical model of the process is presented, and collocated sensor and actuator pairs are used. The process stability is ensured using a controller with appropriate passivity properties. Sensor and actuator location is also discussed. The performance of the ride control system is shown by power spectra of the vertical accelerations obtained from full scale experiments with a $35 \mathrm{~m}$ SES.

\section{Introduction}

Surface Effect Ships (SES) are known to offer a high quality ride in heavy sea states compared to conventional catamarans. However, in low and moderate sea states there are problems with discomfort due to high frequency vertical accelerations induced by resonances in the pressurized air cushion. A high performance ride control system is required in order to achieve satisfactory human comfort and crew workability. To develop such a ride control system it is essential to use a sufficiently detailed dynamic model. Previous ride control systems have been based on the coupled equations of motion in heave and pitch as derived by Kaplan and Davis (1974, 1978), and Kaplan et al. (1981). Their work was based on the assumption that the major part of the wave induced loads from the sea was imparted to the craft as dynamic uniform air pressure acting on the wetdeck, while a minor part of the wave induced loads from the sea was imparted to the craft as dynamic water pressure acting on the side-hulls. This work was further extended by Sørensen et al. $(1992,1993)$ and Sørensen $(1993)$, who included the effect of spatial pressure variations in the air cushion. It was found that acoustic resonances in the air cushion caused by incident sea waves resulted in significant vertical vibrations. A distributed model was derived from a boundary value problem formulation where the air flow was represented by a velocity potential subject to

Received 7 October 1993.

$\dagger$ ABB Corporate Research Norway, P.O. Box 90, N-1361 Billingstad, Norway; email: asgeir@nocrc. abb. no.

$\Varangle$ Department of Engineering Cybernetics, The Norwegian Institute of Technology, 7034 Trondheim, Norway; email: oe(aitk.unit.no.

This paper was presented at the 12th IFAC World Congress, Sydney, 18-23 July 1993, and is reprinted with kind permission from Pergamon Press Ltd, Headington Hill Hall, Oxford OX3 OBW, U.K. 
appropriate boundary conditions on the surfaces enclosing the air cushion volume. A solution was found using the Helmholtz equation in the air cushion region. In this paper the mathematical model presented in Sørensen et al. (1993) is slightly modified and adapted to control system design. This mathematical model is then used to derive a new ride control system. The stability of the control system is analysed using the theory of passivity as presented in Desoer and Vidyasagar (1975) and in Vidyasagar (1993). It is demonstrated that under appropriate assumptions the dynamic system to be controlled is passive, and stability can be achieved using a strictly passive controller.

\section{Mathematical modeling}

In this section a mathematical model for the heave and pitch motion of SES is presented. A detailed derivation of the model is found in Sørensen (1993). A moving coordinate frame is defined so that the origin is located in the mean water plane below the centre of gravity with the $x-, y$-and $z$-axes oriented positive forwards, to the port, and upwards respectively (Fig. 1). Translation along the $z$-axis is called heave and is denoted $\eta_{3}(t)$. The rotation angle around the $y$-axis is called pitch and is denoted $\eta_{5}(t)$. Heave is defined positive upwards, and pitch is defined positive with the bow down. The craft is assumed to be advancing forward in regular head sea waves. The waves are assumed to have a small wave slope with circular frequency $\omega_{o}$. The circular frequency of encounter $\omega_{e}$ is

$$
\omega_{e}=\omega_{o}+k U
$$

where $k=2 \pi / \lambda$ is the wave number, $\lambda$ is the sea wave length and $U$ is the craft speed. The incident surface wave elevation $\zeta(x, t)$ for regular head sea is defined as

$$
\zeta(x, t)=\zeta_{a} \sin \left(\omega_{e} t+k x\right)
$$

where $\zeta_{a}$ is the sea wave elevation amplitude. The beam $b$ and the equilibrium height $h_{o}$ of the air cushion are assumed to be much less than the length $L$. Hence, a onedimensional ideal and compressible air flow in the $x$-direction is assumed. The cushion length is reaching from $x=-L / 2$ at the stern (AP) to $x=L / 2$ at the bow (FP). The
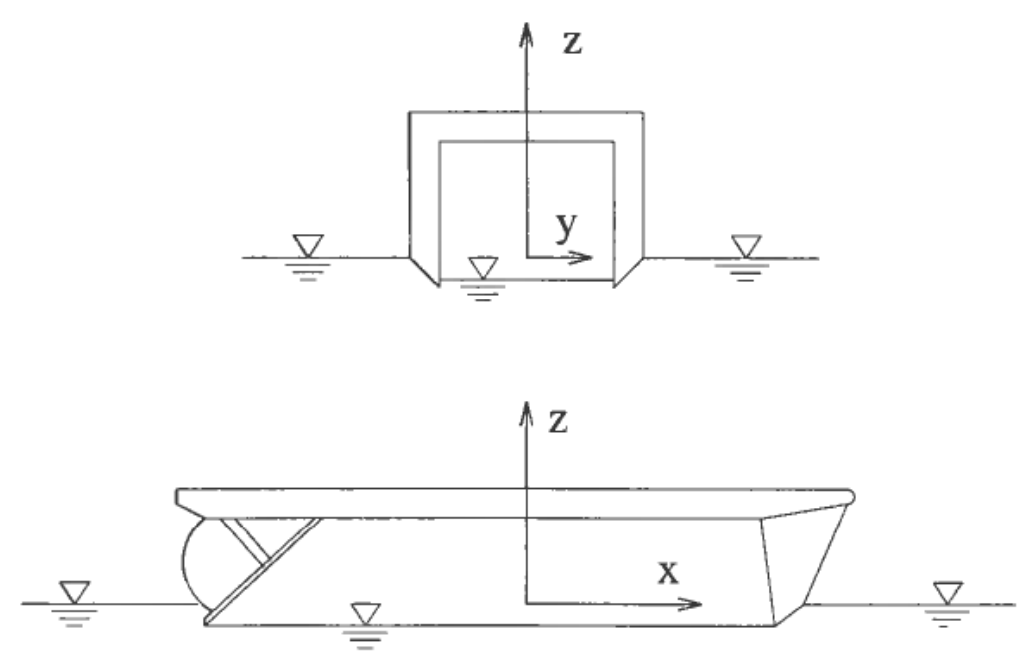

Figure 1. Surface Effect Ship (SES)-coordinate frame. 
longitudinal position of the centre of air cushion pressure is assumed to coincide with the origin of the coordinate frame. The air cushion area is given by $A_{c}=L b$. The total pressure variation $p_{c}(x, t)$, in the air cushion is

$$
p_{c}(x, t)=p_{a}+p_{u}(t)+p_{s p}(x, t)
$$

where $p_{a}$ is the atmospheric pressure, $p_{u}(t)$ is the dynamic uniform excess pressure and $p_{s p}(x, t)$ is the spatially varying pressure. The dynamic air cushion pressure is excited by incoming sea waves. In the absence of sea waves, the stationary excess pressure in the air cushion is equal to the equilibrium excess pressure $p_{o}$. The nondimensional uniform pressure variations $\mu_{u}(t)$ and the nondimensional spatial pressure variations $\mu_{s p}(x, t)$ are defined according to

$$
\mu_{u}(t)=\frac{p_{u}(t)-p_{o}}{p_{o}}, \quad \mu_{s p}(x, t)=\frac{p_{s p}(x, t)}{p_{o}}
$$

The volumetric air flow into the air cushion is given by a linearization of the fan characteristics about the craft equilibrium operating point according to

$$
w_{i n}(t)=\rho_{c o} \sum_{i=1}^{q}\left(Q_{o i}+\left.p_{o} \frac{\partial Q}{\partial p}\right|_{o i}\left(\mu_{u}(t)+\mu_{s p}\left(x_{\mathrm{Fi}}, t\right)\right)\right)
$$

It is assumed that $q$ fans with constant RPM are feeding the cushion where fan $i$ is located at the longitudinal position $x_{F i}, Q_{o i}$ is the equilibrium air flow rate of fan $i$ when $p_{u}(t)=p_{o}, \rho_{c o}$ is the density of the air at $p_{o}$ and $\left.(\partial Q / \partial p)\right|_{o i}$ is the corresponding linear fan slope about the craft equilibrium operating point $Q_{o i}$ and $p_{o}$. The volumetric air flow out of the air cushion is proportional to the leakage area $A_{L}(t)$, which is defined as

$$
A_{L}(t)=A_{o}+A^{R C S}(t)
$$

where $A^{R C S}(t)$ is the controlled variable leakage area, and $A_{o}=A_{o}^{A P}+A_{o}^{A P}$ is the equilibrium leakage area with $A_{o}^{A P}$ defined as the stern equilibrium leakage area at $x=$ $-L / 2$ and $A_{o}^{F P}$ defined as the bow equilibrium leakage area at $x=L / 2$. The controlled leakage area $A^{R C s}(t)$ is written

$$
A^{R C S}(t)=\sum_{i=1}^{r}\left(A_{o i}^{R C S}+\Delta A_{i}^{R C S}\left(x_{s i}, t\right)\right)
$$

where $r$ is the number of louvers, $A_{o i}^{R C S}$ is defined as the mean operating value or bias of the leakage area of louver $i$ which is located at the longitudinal position $x=x_{L i}$. $\Delta A_{i}^{R C S}\left(x_{s i}, t\right)$ is defined as the commanded variable leakage area of louver $i$. A louver is a variable vent valve which changes the area of opening in the wetdeck for the purpose of leakage control. Pressure sensors located at the longitudinal position $x=x_{s i}$, are used to measure the pressure variations in the air cushion.

\subsection{Equations of motion}

In this section the coupled equations of motion in heave and pitch and the dynamic cushion pressure are presented. The dynamic uniform pressure equation is derived from a global continuity equation for the air flow into and out of the air cushion. The spatially varying pressure equation is derived in the time domain from a boundary value problem formulation by solving the wave equation in the air cushion region subject to appropriate boundary conditions on the surfaces enclosing the air cushion volume. 
1. Uniform pressure equation:

$$
K_{1} \dot{\mu}_{u}(t)+K_{3} \mu_{u}(t)+\rho_{c o} A_{c} \dot{\eta}_{3}(t)=K_{2} \sum_{i=1}^{r} \Delta A_{i}^{R C S}\left(x_{s i}, t\right)+\rho_{c o} \dot{V}_{0}(t)
$$

where the constants $K_{1}, K_{2}$ and $K_{3}$ are defined according to

$$
\begin{aligned}
& K_{1}=\frac{\rho_{c o} h_{o} A_{c}}{\gamma\left(1+\left(p_{a} / p_{o}\right)\right)}, \quad K_{2}=\rho_{c o} c_{u}\left(2 p_{o} / \rho_{a}\right)^{1 / 2} \\
& K_{3}=\rho_{c o} \sum_{i=1}^{q}\left(\frac{Q_{o i}}{2}-\left.p_{o} \frac{\partial Q}{\partial p}\right|_{o i}\right)
\end{aligned}
$$

where $\rho_{a}$ is the air density at the atmospheric pressure $p_{a}, \gamma$ is the ratio of specific heat for air and $c_{n}=0.61$ is the orifice coefficient. The time derivative of $V_{o}(t)$ is the wave volume pumping of the dynamic uniform pressure and is found to be

$$
\dot{V}_{0}(t)=b \int_{-L / 2}^{L / 2} \dot{\zeta}(x, t) d x=A_{\mathrm{c}} \zeta_{a} \omega_{e} \frac{\sin (k L / 2)}{k L / 2} \cos \omega_{e} t
$$

2. Spatially varying pressure equation:

$$
\mu_{s p}(x, t)=\sum_{j=1}^{\infty} \dot{p}_{j}(t) r_{j}(x)
$$

where $r_{j}(x)$ is the eigenfunction or the mode shape function of mode $j$, which is found to be

$$
r_{j}(x)=\cos \frac{j \pi}{L}\left(x+\frac{L}{2}\right), \quad x \in\left[-\frac{L}{2}, \frac{L}{2}\right]
$$

The time derivatives of $p_{j}(t)$ is the modal amplitude function for mode $j$. The odd modes around the centre of pressure for $j=1,3,5, \ldots$ is written

where

$$
\begin{aligned}
\ddot{p}_{j}(t)+2 \xi_{j} \omega_{j} \dot{p}_{j}(t)+\omega_{j}^{2}(t) p_{j}(t)=- & c_{2 j} \dot{\eta}_{5}(t) \\
& +c_{1} \sum_{i=1}^{r} \cos \frac{j \pi}{L}\left(x_{L i}+\frac{{ }^{L}}{2}\right) \Delta A_{i}^{R C S}\left(x_{s i} t\right)+\rho_{c o} \dot{V}_{j}(t)
\end{aligned}
$$

$$
c_{1}=\frac{2 K_{2} c^{2}}{p_{o} V_{c o}}, \quad c_{2 j}=\frac{4 \rho_{c o} L c^{2}}{p_{o} \bar{h}_{0}(j \pi)^{2}}
$$

$c$ is the speed of sound in air. The wave volume pumping of the odd acoustic modes is

$$
\dot{V}_{j}(t)=-\frac{4 c^{2}}{p_{0} h_{0} L} \frac{k \cos (k L / 2)}{(j \pi / L)} \omega_{e} \zeta_{a} \sin \omega_{\mathrm{e}} t
$$

The even modes around the centre of pressure $j=2,4,6, \ldots$ is

$$
\ddot{p}_{j}(t)+2 \xi_{j} \omega_{j} \dot{p}_{j}(t)+\omega_{j}^{2} p_{j}(t)=c_{1} \sum_{i=1}^{r} \cos \frac{j \pi}{L}\left(x_{L i}+\frac{L}{2}\right) \Delta A_{i}^{R C S}\left(x_{s i}, t\right)+\rho_{c o} \dot{V}_{j}(t)
$$

where the wave volume pumping of the even acoustic modes is

$$
\dot{V}_{j}(t)=\frac{4 c^{2}}{p_{o} h_{o} L} \frac{k \sin (k L / 2)}{k^{2}-(j \pi / L)} \omega_{e} \zeta_{a} \cos \omega_{e} t
$$


The relative damping ratio $\xi_{j}$ for the odd and even modes is

$$
\xi_{j}=\frac{c}{j \pi h_{o} b}\left(\frac{K_{2}}{2 p_{0}} A_{o}+\frac{K_{2}}{2 p_{o}} \sum_{i=1}^{r} A_{o i}^{R C S} \cos ^{2} \frac{j \pi}{L}\left(x_{L i}+\frac{L}{2}\right)-\left.\rho_{c o} \sum_{i=1}^{q} \frac{\partial Q}{\partial p}\right|_{o i} \cos ^{2} \frac{j \pi}{L}\left(x_{F i}+\frac{L}{2}\right)\right)
$$

and the corresponding eigenfrequency $\omega_{j}$ for the odd and even modes is found to be

$$
\omega_{j}=c \frac{j \pi}{L}, \quad j=1,2,3, \ldots
$$

3. Heave equation:

$$
\left(m+A_{33}\right) \ddot{\eta}_{3}(t)+B_{33} \dot{\eta}_{3}(t)+C_{33} \eta_{3}(t)-A_{c} p_{o} \mu_{u}(t)=F_{3}^{e}(t)
$$

where $m$ is the craft mass.

4. Pitch equation:

$$
\left(I_{55}+A_{55}\right) \ddot{\eta}_{5}(t)+B_{55} \dot{\eta}_{5}(t)+C_{55} \eta_{5}(t)-2 p_{o} b \sum_{j=1,3, \ldots}\left(\frac{L}{j \pi}\right)^{2} \dot{p}_{j}(t)=F_{5}^{e}(t)
$$

where $I_{55}$ is the moment of inertia around the $y$-axis. For simplicity, and without loss of generality, hydrodynamic and hydrostatic coupling like $A_{i j}-B_{i j}$ and $C_{i j}$-terms, and the coupling between the dynamic uniform pressure and the spatially varying pressure is assumed to be negligible and hence is set equal to zero in the model. The hydrostatic $C_{i j}$ - terms are found in the standard way by integration over the water plane area of the side-hulls. The hydrodynamic added-mass coefficients $A_{i i}$, the water wave radiation damping coefficients $B_{i i}$, and the hydrodynamic excitation force in heave $F_{3}^{e}(t)$ and moment in pitch $F_{5}^{e}(t)$, are derived from hydrodynamic loads on the side-hulls (Salvesen 1993). Since the main focus in this paper is on the high frequency range, we have used a simplified strip theory based on Salvesen et al. (1970) for calculation of the hydrodynamic loads.

\subsection{Discussion of the mathematical model}

It is evident from (20) and (21) that the heave and pitch to motions are coupled to the dynamic excess pressure in the air cushion region. This is to be expected since the major part of the SES mass is supported by the air cushion excess pressure. The relative damping ratio $\xi_{j}$ given by $(18)$ is an important parameter. As expected the leakage terms and the fan inflow term contribute to increased damping. One should notice that the fan slope $\left.(\partial Q / \partial p)\right|_{o}$, is negative. The longitudinal location of the fan and the louver systems strongly affects the relative damping ratio. In the case of a single fan system and a single louver system, it may seem natural to locate the fan and the louver in the middle of the air cushion, that is $x_{F}=x_{L}=0$. However, from (18) we observe that the relative damping ratio for the odd modes will be reduced significantly if $x_{L}$ and $x_{F}$ are equal to 0 . Maximum damping of both the odd and even acoustic resonance modes is obtained for $x_{F}$ and $x_{L}$ equal to $-L / 2$ or $L / 2$. The relative damping ratio of the first odd acoustic mode on a $35 \mathrm{~m}$ SES with the ride control system off, will increase from about 0.05 to 0.2 by locating the lift fan system at one of the ends of the air cushion instead in the middle. This gives a significant improvement in ride quality even when the ride control system is turned off. In the same manner, the active damping due to the ride control system is maximized by locating the louver system at one of the ends of the air cushion. In the following we will use a finite number of $k$ acoustic modes in the mathematical model. The effect of higher order modes is assumed to be negligible. 


\section{Robust dissipative controller design}

In this section a ride control system based on the mathematical model derived in the previous section is developed. The objective of the control system is to damp out pressure fluctuations around the equilibrium pressure $p_{o}$ in the presence of sea wave disturbances. This can be formulated in terms of the desired value of the nondimensional dynamic uniform pressure $\mu_{u}^{d}(t)=0$ and the nondimensional spatially varying pressure $\mu_{s p}^{d}(x, t)=0$, where the superscript $d$ denotes the desired value. The number of modes to be damped depends on the requirements related to established criteria for human comfort and working conditions for crew. The mathematical model of the craft dynamics is of high order as it contains a high number of acoustic modes. A practically implementable controller has to be of reduced order. When designing a controller based on a reduced order model, it may happen that the truncated or residual modes result in degradation of the performance, and even instability of the closed loop system. This is asalogous with the so-called spillover effect in active damping of vibrations in mechanical structures (Balas, 1978). This problem was also discussed by Gevarter (1970) in connection with the control of flexible vehicles. The controller must be robust with respect to modeling errors and parametric and non-parametric uncertainties, nonlinearities in sensors and actuators; and component failure. The use of collocated compatible actuator and sensor pairs provides a design technique for circumventing these problems. In this case a passive model description can be obtained, and robust stability can be achieved by using a controller with appropriate passivity properties.

\subsection{State space model}

The dynamic system given by (8)-(21) is of third order as opposed to similar vibration damping problems of large flexible space structures that can be written as equivalent second order mass, damper and spring system, see e.g. Joshi (1989). In this paper the theory of passivity as presented in Desoer and Vidyasagar (1975) and Vidyasagar (1993) is used to analyse stability. The dynamic system given by the (8)-(21) is written in standard state space form

$$
\begin{aligned}
& \dot{\boldsymbol{x}}=A \boldsymbol{x}+B \boldsymbol{u}+E \boldsymbol{v} \\
& \boldsymbol{y}=C \boldsymbol{x}
\end{aligned}
$$

where the $n$ dimensional state vector $\mathbf{x}(t)$ is

$$
\boldsymbol{x}=\left[\eta_{3}, \eta_{5}, \dot{\eta}_{3}, \dot{\eta}_{5}, \mu_{w}, p_{1}, \ldots, p_{k}, \dot{p}_{1}, \ldots, \dot{p}_{k}\right]^{\mathrm{T}}
$$

where $\eta_{3}(t), \eta_{5}(t), \mu_{u}(t), p_{1}(t), p_{2}(t), \ldots, p_{k}(t)$ are defined as in Section 2.1. $k$ is the number of acoustic modes. $v(t)$ is the $3+k$ dimensional disturbance vector defined as

$$
v(t)=\left[F_{3}^{e}, F_{5}^{e}, \dot{V}_{0}, V_{1}, \dot{V}_{k}\right]^{\mathrm{T}}
$$

where $F_{3}^{e}(t)$ and $F_{5}^{e}(t)$ are defined as in Salvesen (1993). The time derivatives of $V_{0}(t)$, $V_{1}(t), \ldots, V_{k}(t)$ are defined in the (10), (15) and (17). $u(t)$ is the $r$ dimensional control input vector, and $r$ is the number of louvers. The elements of $u(t)$ are for $i=1,2, \ldots, r$ defined as

$$
u_{i}(t)=\Delta A_{i}^{R C s}\left(x_{s i}, t\right)
$$

where $\Delta A_{i}^{R C S}\left(x_{s i}, \mathrm{t}\right)$ is defined in (7). The louver and sensor pairs may be distributed along the air cushion, preferentially in the longitudinal direction. $\mathbf{y}(t)$ is the $m$ dimensional measurement vector and $m$ is the number of pressure sensors. The symbolic expressions for the $n \times n$ system matrix $A, n \times r$ control input matrix $B$, 
$n \times(3+k)$ disturbance matrix $E$ and $m \times n$ measurement matrix $C$ can be derived in a standard manner from the equations of motion presented in Section 2.1. Consider the case where the sensors and actuators are ideal, that is linear and instantaneous with no noise. It is assumed that the control input matrix $B$ can be chosen such that

$$
C=B^{\mathrm{T}} \mathrm{P}
$$

where $P$, which is given in (34), is a $n \times n$ diagonal positive definite matrix providing correct scaling of the $B^{\mathrm{T}}$ matrix to the $C$ matrix. This is referred to the case when there is perfect collocation between the sensors and the louvers, i.e. $x_{L i}=x_{s i}$ for all $i$ and $r=m$. We can derive linear time-invariant operators between the inputs and the outputs of the dynamic system given by (22). Let $s$ be the differential operator. It can be shown that the pair $(A, B)$ is controllable and the pair $(A, C)$ is observable. Hence, the dynamic system given by (22) can be represented by

$$
\boldsymbol{y}(s)=H_{p}(s) \boldsymbol{u}(s)+H_{d}(s) v(s)=\boldsymbol{y}_{u}(s)+\boldsymbol{y}_{v}(s)
$$

where the transfer matrices $H_{p}(s)$ and $H_{d}(s)$ are given by

$$
\begin{aligned}
& H_{p}(s)=C\left(s I_{n}-A\right)^{-1} B \\
& H_{d}(s)=C\left(s I_{n}-A\right)^{-1} E
\end{aligned}
$$

and $I_{n}$ is the $n \times n$ identity matrix.

\subsection{Stability properties of the control system}

In this section a strictly passive controller with finite gain is proposed. Employing the definitions of passivity as presented in Desoer and Vidyasagar (1975), and in Vidyasagar (1993), on an interconnected system consisting of two subsystems in a standard feedback configuration (Fig. 2), the robust stability of the feedback system can be shown for certain input-output properties of the subsystems. The following lemma showing that the $n \times n$ system matrix $A$ is Hurwitz will then be utilized.

\section{Lemma 1}

The eigenvalues of $n \times n$ system matrix A given in (22) have negative real parts.

Proof:

Consider the autonomous system of (22) with $\mathbf{u}(t)=\mathbf{v}(t)=0$. Define the Lyapunov function candidate

$$
V(x)=\frac{1}{2} x^{\mathrm{T}} P x>0
$$

where the $n \times n$ diagonal positive definite matrix $P$ is given in (34), $\boldsymbol{V}(\boldsymbol{x})$ is positive definite.

The time derivative of $V(x)$ along system trajectories is

$$
\dot{V}(\mathbf{x})=\frac{1}{2} \mathbf{x}^{\mathrm{T}}\left(A^{\mathrm{T}} P+P A\right) \mathbf{x}=-\frac{1}{2} \mathbf{x}^{\mathrm{T}} Q \mathbf{x} \leqslant 0
$$

The $n \times n$ diagonal positive semidefinite matrix $Q$ is

$$
Q=\operatorname{diag}\left[0_{2 \times 2}, Q 1_{3 \times 3}, 0_{k \times k}, Q 2_{k \times k}\right]
$$


The $3 \times 3$ diagonal positive definite $Q 1_{3 \times 3}$ matrix is

$$
\begin{gathered}
Q 1_{3 \times 3}=\operatorname{diag}\left[q_{i i}\right], i=3,4,5 \\
q_{33}=P_{3} \frac{2 B_{33}}{m+A_{33}}, \quad q_{44}=P_{4} \frac{2 B_{55}}{I_{55}+A_{55}}, \quad q_{55}=P_{5} \frac{2 K_{3}}{K_{1}}
\end{gathered}
$$

The $k \times k$ diagonal positive definite $Q 2_{k \times k}$ matrix is

$$
\begin{gathered}
Q 2_{k \times k}=\operatorname{diag}\left[q_{i i}\right], i=5+k+1,5+k+2, \ldots, 5+2 k \\
q_{(5+k+j)(5+k+j)}=P_{(5+k+j)(5+k+j)} 4 \xi_{j} \omega_{j}, j=1,2,3, \ldots, k
\end{gathered}
$$

The $n \times n$ diagonal positive definite matrix $P$ is found from (30) to be

$$
\begin{aligned}
P & =\operatorname{diag}\left[P_{i}\right], \quad i=1,2,3, \ldots, n, \quad j=1,2,3, \ldots, k \\
P_{1} & =P_{3} \frac{C_{33}}{m+A_{33}}, \quad P_{2}=P_{4} \frac{C_{55}}{I_{55}+A_{55}}, \quad P_{3}=P_{5} \frac{\rho_{c o}\left(m+A_{33}\right)}{K_{1} p_{0}} \\
P_{4} & =-\frac{g_{1}}{d_{1} c_{1}}, \quad P_{5}=\frac{K_{1}}{\rho_{c o} K_{2}}, \quad P_{5+j}=\omega_{j}^{2} P_{5+k+j}, \quad P_{5+k+j}=\frac{1}{c_{1}}
\end{aligned}
$$

where $c_{1}$ is defined in (14), From (22) and (30) it is seen that

$$
\begin{gathered}
\dot{V}(\mathbf{x})=0 \\
\Downarrow \\
\boldsymbol{x}=\boldsymbol{x}_{o}=\left[\eta_{3}, \eta_{5}, 0,0,0, p_{1}, p_{2}, \ldots, p_{k}, 0,0, \ldots, 0\right]^{\mathrm{T}}
\end{gathered}
$$

However, from (22)

$$
\ddot{\eta}_{3}=\ddot{\eta}_{5}=\dot{\mu}_{u}=\ddot{p}_{1}=\ddot{p}_{2}=\ldots=\ddot{p}_{k}=0
$$

which implies

$$
\eta_{3}=\eta_{5}=p_{1}=p_{2}=\ldots=p_{k}=0
$$

Hence, by the invariant set theorem (Vidyasagar, 1993) the equilibrium point $x_{o}=0$ is asymptotically stable and the result of Lemma 1 follows.

Define the linear time-invariant operators $H_{p}: L_{2 e}^{m} \rightarrow L_{2 e}^{r}(r=m)$ and assume that $H_{d}$ : $L_{2}^{3+k} \leftarrow L_{2}^{m}$, such that $y_{v} \in L_{2}^{3+k}$ whenever $v \in L_{2}^{3+k}$. Notice that $L_{2 e}$ is the extended space of $L_{2}$ (Desoer and Vidyasagar, 1975). In the following lemma it is shown that the process operator $H_{p}$ is passive. This allows for the design of robust, stable output feedback controllers for ride control of SES.

\section{Lemma 2}

The process operator $H_{p}$ is passive.

\section{Proof}

Set $v(t)=0$ in (22) and use the Lyapunov function candidate as given in (29). $V(x)$ is positive definite. The time derivative of $V(x)$ along the system trajectories is

$$
\dot{V}(x)=\frac{1}{2} x^{\mathrm{T}}\left(A^{\mathrm{T}} P+P A\right) \boldsymbol{x}+\boldsymbol{x}^{\mathrm{T}} P B \boldsymbol{u}
$$


The $n \times n$ diagonal positive semidefinite matrix $Q$ is given in (31). If we assume perfect collocation between the sensor and actuator pairs, that is $C=B^{\mathrm{T}} P,(38)$ becomes

$$
\begin{aligned}
\dot{V}(\boldsymbol{x}) & =x^{\mathrm{T}} C^{\mathrm{T}} \boldsymbol{u}-\frac{1}{2} \boldsymbol{x}^{\mathrm{T}} Q \mathbf{x} \\
& =\boldsymbol{y}_{u}^{\mathrm{T}} \boldsymbol{u}-\frac{1}{2} \boldsymbol{x}^{\mathrm{T}} Q \boldsymbol{x}
\end{aligned}
$$

Integrating (39) from $t=0$ to $t=T$ we obtain

$$
\left(y_{u}, u\right)_{\mathrm{T}}=V(t=T)-V(t=0)+\frac{1}{2} \int_{0}^{\mathrm{T}} x^{\mathrm{T}} Q x \mathrm{~d} t
$$

Since $Q \geqslant 0$ and $V(t=T)>0$, (40) can be written

$$
\left(y_{u}, u\right)_{\mathrm{T}} \geqslant-V(t=0) \triangleq \beta
$$

and the result of Lemma 2 follows

\section{Remark 1}

It is evident from (22) that if the initial conditions are equal to zero that is $x(t=0)$ $=0$, then $\beta=-\frac{1}{2} \mathbf{x}^{\mathbf{T}}(t=0) P \mathbf{x}(t=0)=0$.

\section{Remark 2}

The transfer matrix $H_{d}(s)$ of the linear time-invariant operator $H_{d}$ as defined by (28) is strictly proper and all the poles have negative real parts according to Lemma 1. Hence, if $v \in L_{2}^{3+k}$, then $y=H_{d} v \in L_{2}^{m} \cap L_{\infty}^{m}$.

Let the controller be defined as the linear time-invariant operator $H_{c}$ between the input $\mathbf{y}=\mathbf{y}_{u}+\boldsymbol{y}_{v}$ and the output $\boldsymbol{u}_{c}$. Connecting the $H_{c}$ operator with the $H_{p}$ and $H_{d}$ operators, we obtain the feedback system illustrated in Fig. 2. The transfer matrix of $H_{c}$ is denoted $H_{c}(s)$.

\section{Proportional control law}

A strictly passive proportional pressure feedback controller of dimension $r \times r$ with finite gain is proposed according to

$$
\begin{aligned}
\boldsymbol{u}_{c}(s) & =H_{c}(s) y(s) \\
H_{c}(s) & =G_{p}
\end{aligned}
$$

where $G_{p}=\operatorname{diag}\left[g_{p i}\right]>0$ is a constant diagonal feedback gain matrix of dimension $r \times r$. This control law provides enhanced damping of the pressure variations around the resonance frequencies.

The main result of this section is contained in the following theorem.

\section{Theorem 1}

Consider the following feedback system (see, Fig. 2)

$$
\begin{aligned}
\boldsymbol{y}_{u} & =H_{p} \boldsymbol{u} \\
\boldsymbol{y}_{\boldsymbol{v}} & =H_{d} \boldsymbol{v} \\
\boldsymbol{y} & =\boldsymbol{y}_{\boldsymbol{u}}+\boldsymbol{y}_{\boldsymbol{v}} \\
\boldsymbol{u} & =-\boldsymbol{u}_{\boldsymbol{c}}=-\boldsymbol{H}_{c} \boldsymbol{y}
\end{aligned}
$$

where $H_{p}, H_{\mathrm{c}}: L_{2 e}^{m} \rightarrow L_{2 e}^{m}$. Assume that $H_{d}: L_{2}^{3+k} \rightarrow L_{2}^{m}$, so that $y_{v} \in L_{2}^{m}$ whenever $v \in L_{2}^{3+k}$. $H_{c}$ is strictly passive with finite gain and $H_{p}$ is passive. Hence, the feedback system defined in (43) is $L_{2}^{m}$ stable and since the feedback system given by $H_{p}, H_{p}$ and $H_{c}$ is linear, $L_{2}^{m}$ stability is equivalent to $L_{\mathrm{o}}^{m}(\mathrm{BIBO})$ stability. 
Proof

Set $v(t)=0$ in (22) and use the Lyapunov function candidate as given in (29). $V(x)$ is positive definite. If we assume perfect collocation between the sensor and actuator pairs, that is $C=B^{\mathrm{T}} P$. The time derivative of $V(x)$ along the closed loop system trajectory becomes

$$
\begin{aligned}
\dot{V}(\mathbf{x}) & =-y_{u}^{\mathrm{T}} G_{p} \mathbf{y}_{u}-\frac{1}{2} \mathbf{x}^{\mathrm{T}} Q \mathbf{x} \\
& =-\mathbf{x}^{\mathrm{T}}\left(C^{\mathrm{T}} G_{p} C+\frac{1}{2} Q\right) \boldsymbol{x}
\end{aligned}
$$

where the $n \times n$ diagonal positive semidefinite matrix $Q$ is given in (31). It can be demonstrated by inspection that

$$
C^{\mathrm{T}} G_{p} C+\frac{1}{2} Q \geqslant 0
$$

since the first term in (45) is on quadratic form for diagonal $G_{p}>0$ and hence positive semidefinite. The time derivative of $\boldsymbol{V}(\boldsymbol{x})$ is negative semidefinite. Using the invariant set theorem (Vidyasagar, 1993) the equilibrium point of the closed loop system is asymptotically stable and the result follows.

$L_{2}^{m}$ and $L_{\infty}^{m}$ stability of the closed-loop system using collocated sensor and actuator pairs is maintained regardless of the number of modes, and regardless of the inaccuracy in the knowledge of the parameters. Thus the spillover problem is eliminated and the parameters do not have to be known in advance for stability to be obtained. Notice that there are no restrictions on the location of the collocated sensor and actuator pairs with respect to stability. However, for optimizing the performance, the longitudinal location of the sensor actuator pairs is crucial, as seen in (18). Robustness with respect to unmodeled dynamics and sector nonlinearities in the actuators are shown in Sørensen (1993). It is further shown by Sørensen (1993) that some imprecision in the collocated sensor and actuator pairs can be accepted without violating the stability properties of the closed-loop system.

\section{Simulation and full scale results}

In this section numerical simulations and results from full scale trials with a $35 \mathrm{~m}$ SES advancing at high speed in head sea waves are presented. The effect of collocation and noncollocation of the sensor and actuator pairs for the $35 \mathrm{~m}$ SES is investigated. The SES is equipped with one single fan and louver system. Main dimensions and data of the SES craft are given in Appendix A. The number of acoustic modes considered in the simulation model is four, i.e. $k=4$.

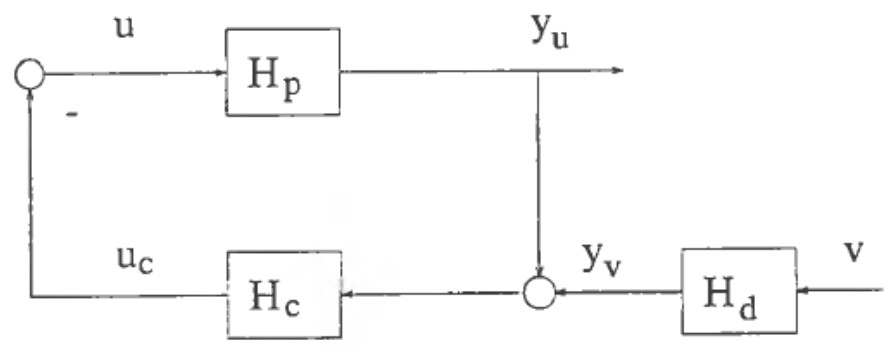

Figure 2. Feedback system. 

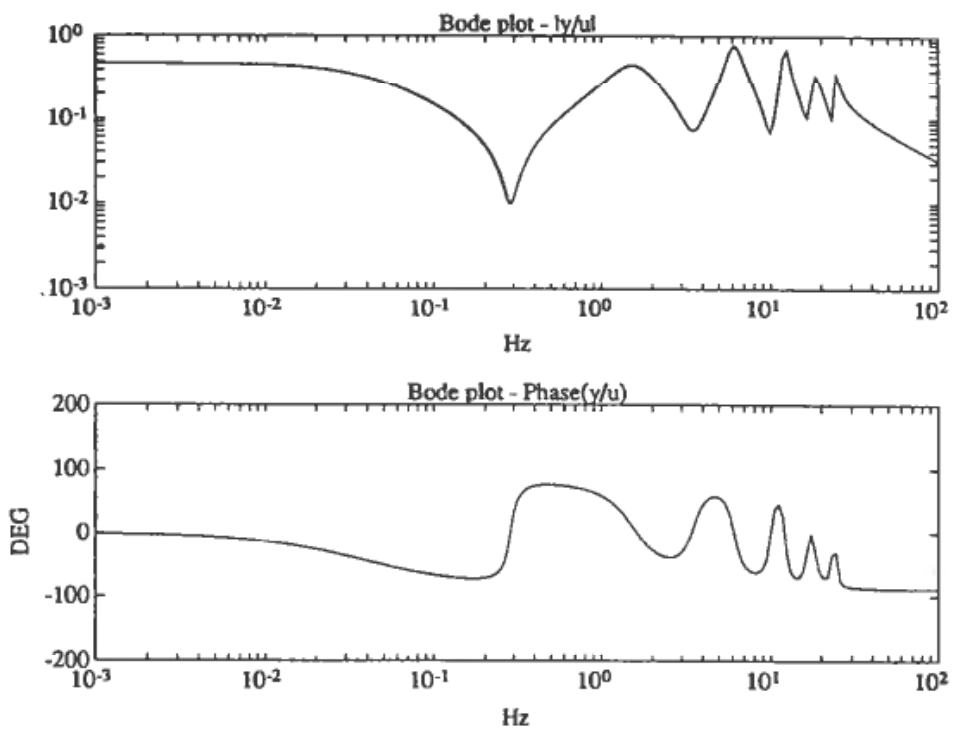

Figure 3. Num. calc. Bode plot of $H_{p}\left(i \omega_{e}\right) ; x_{L}=x_{s}=12 \mathrm{~m}, x_{F}=6 \mathrm{~m}, U=50$ knots, $p_{o}=500 \mathrm{mmWc}$.
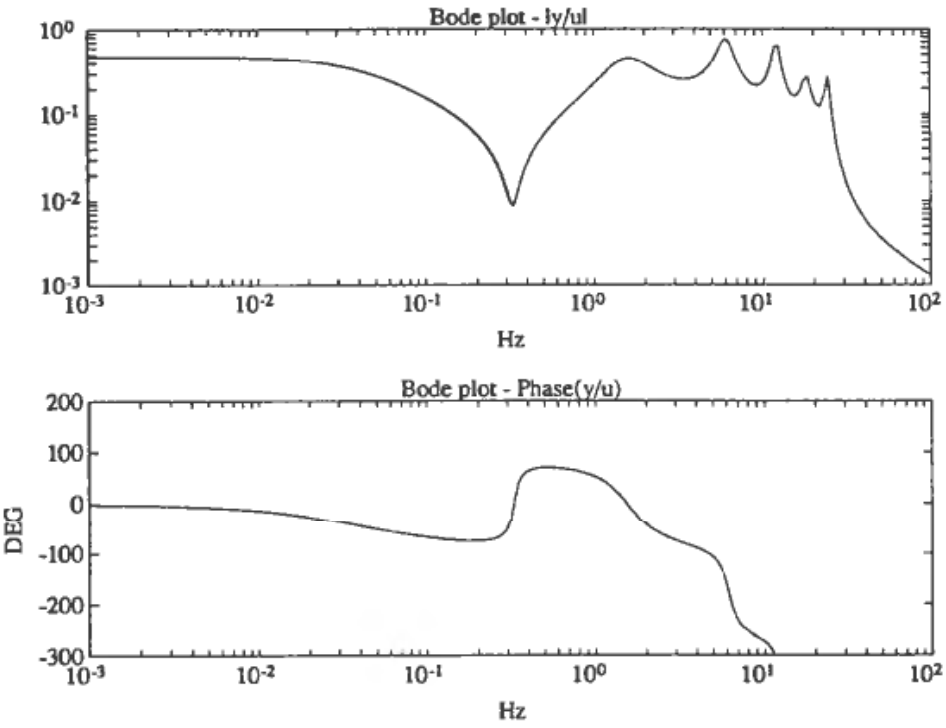

Figure 4. Num. calc. Bode plot of $H_{p}\left(i \omega_{c}\right) ; x_{s}=12 \mathrm{~m}, x_{L}=-12 \mathrm{~m}, x_{F}=6 \mathrm{~m}, U=50 \mathrm{knots}$. $p_{o}=500 \mathrm{mmWc}$. 


\subsection{Numerical simulations}

Fig. 3 shows the Bode plot of $H_{p}\left(i \omega_{e}\right)$ between the pressure sensor $y_{u}(s)$ and the louver $u(s)$ when the pressure sensor and actuator pair is fully collocated. The sensor and louver pair is located at the fore end of the air cushion. When the frequency of encounter goes to zero, the dynamic pressure tends to be a static value proportional to $K_{1} / K_{2}$. This indicates that the equilibrium pressure $p_{o}$ will decrease when the equilibrium leakage area increases and vice versa. Around $0-1 \mathrm{~Hz}$ the response is close to zero. This is related to the structural mass forces acting on the SES and the hydrodynamic forces acting on the side-hulls. The high value around $2 \mathrm{~Hz}$ is due to the resonance of the dynamic uniform pressure. The high values around $6 \mathrm{~Hz}, 12 \mathrm{~Hz}, 18 \mathrm{~Hz}$ and $24 \mathrm{~Hz}$ are related to the four acoustic resonance modes. From the phase plot we observe that the phase varies between $90^{\circ}$ to $-90^{\circ}$ in the whole frequency range. This is to be expected when using collocated sensor and actuator pairs.

Fig. 4 shows the Bode plots of $H_{p}\left(i \omega_{e}\right)$ when the pressure sensor is located at the fore end of the air cushion while the louver is located at the aft end of the air cushion. From the phase plot we observe that from $6 \mathrm{~Hz}$ and upwards the sensed pressure signal at the fore end is more than $180^{\circ}$ out of phase compared to the pressure signal at the aft end where the louver is located. This is to be expected with noncollocated sensor and louver pairs. Noncollocated sensor and actuator pairs introduce negative phase and leads to instability.

\subsection{Full scale results}

The prototype ride control system used in the full scale experiments was based on the passive controller as presented in Section 3. The control algorithms in the ride control system were partly implemented on a personal computer (PC). Analogue hardware devices were also used. An outer feedback loop was implemented on the PC, while a faster inner feedback loop around the electro-hydraulic louver system was implemented by means of analogue hardware devices. The louver system consisted of two vent valves located side by side at the same longitudinal position $x_{L}=8 \mathrm{~m}$. The two vent valves were operated in parallel in the outer feedback loop. Two pressure sensors located at $x_{s 1}=10 \mathrm{~m}$ and $x_{\mathrm{s} 2}=-10 \mathrm{~m}$ were used to measure the excess pressure variations in the air cushion. One accelerometer located about $5 \mathrm{~m}$ aft of the centre of gravity was used to measure the vertical accelerations. The inner analogue controller loop around the louver system provided the necessary opening and closing actions of the vent valves. The experimental arrangement is illustrated in Fig. 5.

The full scale measurements were carried out in sea states with significant wave heights estimated to vary between $0 \cdot 3-0.6 \mathrm{~m}$. The power spectra of the vertical accelerations with and without the ride control system are presented.

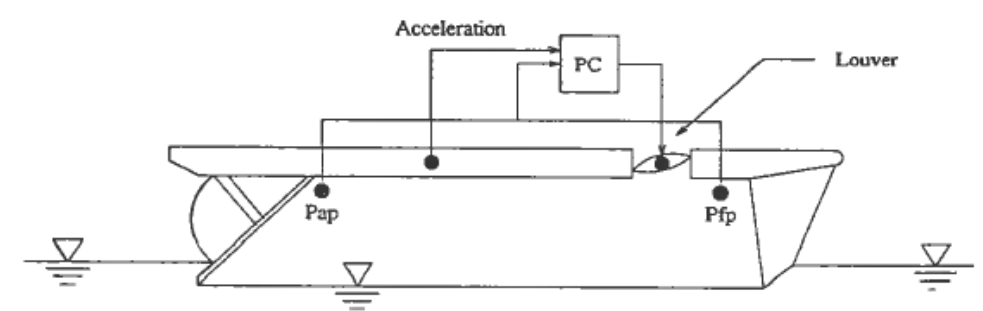

Figure 5. Experiment arrangement. 
$\left[\frac{m}{s^{2}}\right]^{2} s$

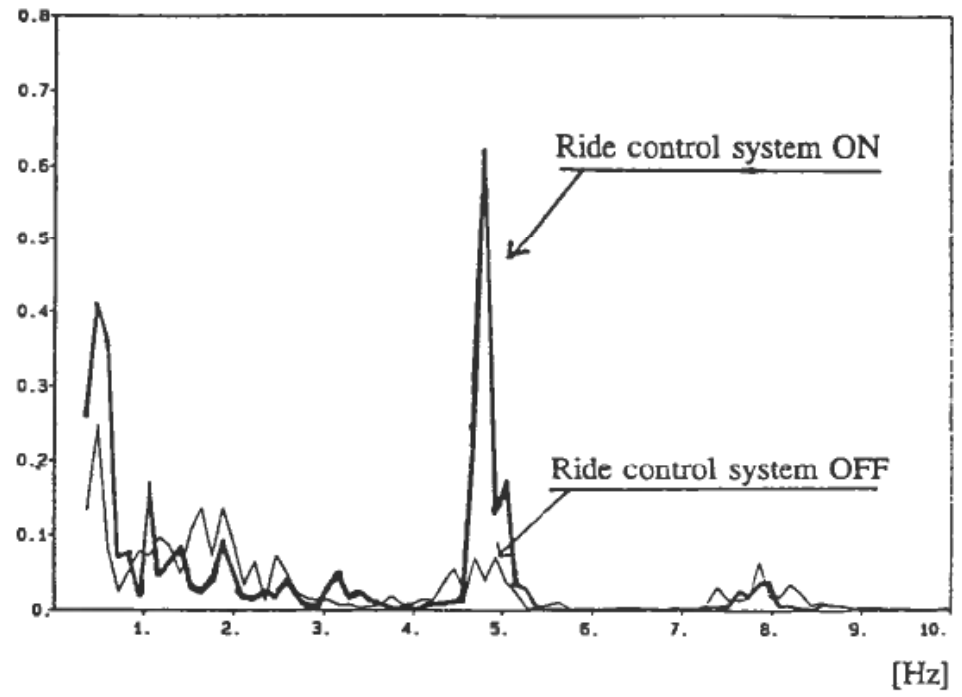

Figure 6. Full scale power spectra of the vertical accelerations at $x=-5 \mathrm{~m}$ of a $35 \mathrm{~m}$ SES with ride control system on and off; $p_{o}=450 \mathrm{mmWc}$.

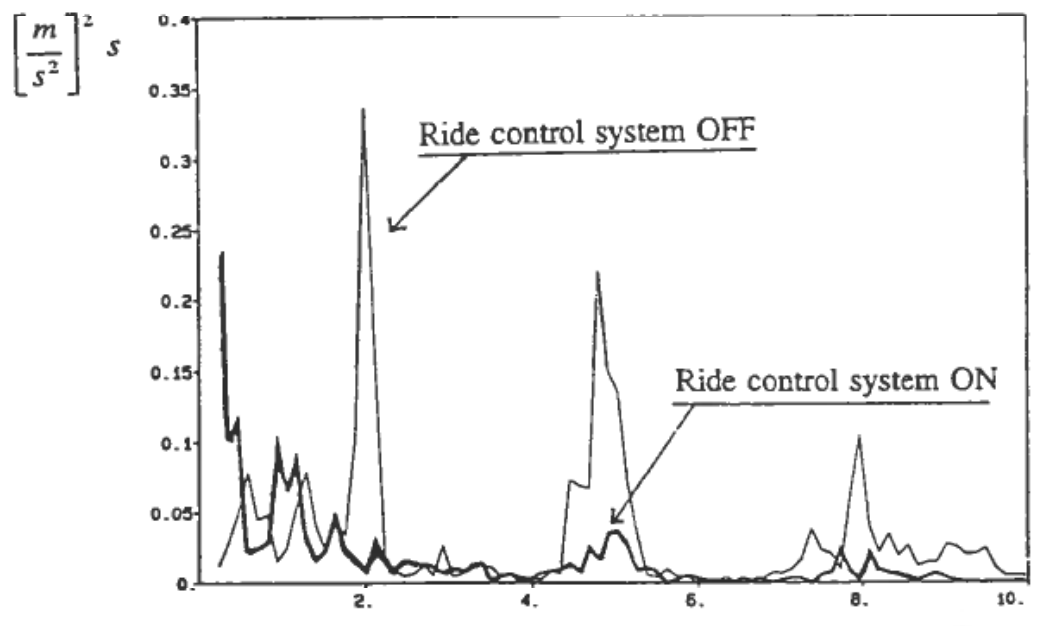

$[\mathrm{Hz}]$

Figure 7. Full scale power spectra of the vertical accelerations at $x=-5 \mathrm{~m}$ of a $35 \mathrm{~m}$ SES with ride control system on and off; $p_{o}=430 \mathrm{mmWc}$.

Fig. 6 shows the full scale power spectra of the vertical accelerations $5 \mathrm{~m}$ aft of the centre of gravity with and without the ride control system activated. With the ride control system turned off, we observed significant response around $2 \mathrm{~Hz}, 5 \mathrm{~Hz}$ and $8 \mathrm{~Hz}$. The response around $2 \mathrm{~Hz}$ is related to the resonance of the dynamic uniform pressure, while the response around $5 \mathrm{~Hz}$ and $8 \mathrm{~Hz}$ is related to the first odd and even resonance modes. When activating the ride control system, the response around $5 \mathrm{~Hz}$ was significantly amplified, while the response around $2 \mathrm{~Hz}$ was only slightly reduced. In 
this case the pressure signal at $x_{s 2}=-10 \mathrm{~m}$ was used in the feedback loop. Hence, the actuator and sensor pair was completely noncollocated since the louver was located at $x_{L}=8 \mathrm{~m}$. This means that for the first odd mode, the noncollocation resulted in positive feedback for this particular mode because the pressure at the sensor location was $180^{\circ}$ out of phase compared to the pressure at the actuator location in the frequency range dominated by the first odd acoustic resonance mode. The results around $8 \mathrm{~Hz}$ was more or less unchanged. Both time series were recorded when the craft was advancing with the speed $U=45 \mathrm{knots}$ in head sea waves with significant wave height estimated to be $H_{s}=0 \cdot 3 \mathrm{~m}$.

Fig. 7 shows the full scale power spectra of the vertical accelerations $5 \mathrm{~m}$ aft of the centre of gravity with and without the ride control system activated. In this case the pressure signal at $x_{s 1}=10 \mathrm{~m}$ was used in the feedback loop. Hence, the louvers and sensors were almost' collocated since the louvers were located at $x_{L}=8 \mathrm{~m}$. With the ride control system turned off, we observed responses around $2 \mathrm{~Hz}, 5 \mathrm{~Hz}$ and $8 \mathrm{~Hz}$. Activating the ride control system, the response around all three resonance frequencies was significantly reduced. These time series were recorded when the craft was advancing with the speed $U=44 \mathrm{knots}$ in head sea waves with significant wave height estimated to be $H_{s}=0.6 \mathrm{~m}$. As seen from fig. 6 and 7 the first and second acoustic resonances appeared at $5 \mathrm{~Hz}$ and $8 \mathrm{~Hz}$ in the full scale experiments. From the mathematical model they were assumed to be $6 \mathrm{~Hz}$ and $12 \mathrm{~Hz}$ respectively. The reason for this reduction of resonance frequencies is the flexibility of the aft seal system, Steen (1993). Flexible seals at the fore and after end of the air cushion mean that the effective air cushion length increases compared to a SES equipped with rigid seals. Hence, from (19) it is seen that the resonance frequency decreases.

\section{Conclusions}

The pressure variations in the pressurized air cushion of a SES have two fundamental characteristics; a dynamic uniform and a spatially varying pressure term. It has been demonstrated that the resonances of the dynamic uniform pressure and the spatially varying pressure cause excessive vertical accelerations when the craft is advancing in sea states which contain energy in the frequency domains corresponding to the resonance frequencies. In order to achieve high human comfort and crew workability, it is necessary to reduce these accelerations using a ride control system. A distributed ride control system has been developed based on the theory of passive systems, and a proportional pressure feedback controller has been proposed. Full scale experiments of a prototype ride control system showed significant improvement in ride quality when using a ride control system which provided dissipation of energy around the resonance frequencies. The full scale experiments also showed the importance of using collocated sensor and actuator pairs in the acoustic dominated frequency range. Spillover effects such as unwanted excitation of residual modes were avoided, regardless of the number of modes considered and the parameter values, through the use of collocated sensor and actuator pairs.

\section{ACKNOWLEDGMENT}

This work has been sponsored by the Ulstein Group and the Royal Norwegian Council for Scientific and Industrial Research (NTNF). The authors are grateful to Dr. Ole J. Sørdalen, Dr. Sverre Steen, Dr. Thor I. Fossen and Dr. Odd M. Faltinsen at the Norwegian Institute of Technology for valuable discussions. 
Length overail

APPENDIX A. SES main dimensions.

Equilibrium fan flow rate

$35 \mathrm{~m}$

Linear fan slope

Cushion length

$150 \mathrm{~m}^{3} / \mathrm{s}$

Nom. cushion pressure

Cushion beam

$-140 \mathrm{~m}^{2} / \mathrm{s}$

$28 \mathrm{~m}$

Cushion height

$500 \mathrm{mmWc}$

$8 \mathrm{~m}$

$2 \mathrm{~m}$

Weight

150 ton

Max speed

50 knots

\section{REFERENCES}

Balas, M. (1978, Feedback control of flexible systems. IEEE Trans. of Automatic Control, 23, 673-679.

Desoer, C. A. and Vidyasagar, M. (1975). Feedback Systems: Input-Output Properties (Academic Press, NY).

FaltinSEN, O. M. (1990), Sea Loads on Ships and Offshore Structures (Cambridge University Press).

Gevarter, W. B. (1970), Basic relations for control of flexible vehicles. AI AA Journal, 8, No. 4.

Joshi, S. M. (1989), Control of Large Flexible Space Structures (Berlin Springer-Verlag).

KAPLAN, P. and DAVIS, S. (1974), A simplified Representation of the Vertical Plane Dynamics of SES Craft. AIAA Paper N. 74-314, AIAA/SNAME Advanced Marine Vehicles Conference, San Diego, California.

KaPI.AN, P. and Davis, S. (1978). System Analysis Techniques for Designing Ride Control System for SES craft in Waves. 5th Ship Control System Symposium, Annapolis, MD (United States Naval Academy, Annapolis).

Kaplan, P., Bentson, J., and Davis, S. (1981), Dynamics and hydrodynamics of surface effect ships. Trans. SNAME, 89, 211-247.

Salvesen, N., Tuck, E. O., and Faltensen, O. M. (1970), Ship motions and sea loads. Trans. SNAME, 78, 345-356.

STEEN, S. (1993). Cobblestone Effect on SES, Dr. ing. Thesis, Depart. of Marine Hydrodynamics, The Norwegian Institute of Technology, Trondheim, Norway.

SORENSEN, A. J. (1993). Modelling and Control of SES Dynamics in the Vertical Plane. Dr. ing. Thesis, Department of Engineering Cybernetics, the Norwegian Institute of Technology.

Sørensen, A. J., Steen, S. and Faltinsen, O. M., (1992). Cobblestone Effect on SES. High Performance Marine Vehicle Conference- HPMV 92 , ASNE, Washington D.C.

SøRENSEN, A. J., STEEN, S., and FALTinSEN, (1993). SES dynamics in the vertical plane. Journal of Ship Technology Research, 40, 71-94.

Vidyasagar, M. (1993), Nonlinear System Analysis. Second Edition (Prentice Hall, Englewood Cliffs, NJ). 